\title{
An enduring error
}

\author{
Branko Grünbaum \\ Branko Grünbaum received his Ph.D. at the Hebrew University in Jerusalem, Israel, \\ in 1957, after emigration with his family from Yugoslavia in 1949. Since then he held \\ professorships at the Hebrew University and several universities in the U.S., the last \\ one being the University of Washington in Seattle, where he is a Professor Emeritus \\ since the year 2000. His major fields of interest are geometry and combinatorics.
}

\section{Introduction}

Mathematical truths are immutable, but mathematicians do make errors, especially when carrying out non-trivial enumerations. Some of the errors are "innocent" - plain mistakes that get corrected as soon as an independent enumeration is carried out. For example, Daublebsky [14] in 1895 found that there are precisely 228 types of configurations (123), that is, collections of 12 lines and 12 points, each incident with three of the others. In fact, as found by Gropp [19] in 1990, the correct number is 229. Another example is provided by the enumeration of the uniform tilings of 3-dimensional space by Andreini [1] in 1905; he claimed that there are precisely 25 types. However, as shown [20] in 1994, the correct number is 28 . Andreini listed some tilings that should not have been included, and missed several others - but again, these are simple errors easily corrected.

Much more insidious are errors that arise by replacing enumeration of one kind of object by enumeration of some other objects - only to disregard the logical and mathematical

Archimedische Körper sind konvexe Polyeder, deren Seitenflächen regelmässige Polygone sind und bei denen die zyklische Anordnung der Seitenflächen um jede Ecke gleich ist. Es stellt sich heraus, dass es neben den fünf Platonischen Körpern und den beiden unendlichen Familien von Prismen und Antiprismen genau vierzehn weitere Archimedische Körper gibt. Der fortwährende Irrtum (,enduring error"), auf den der Titel der Arbeit hinweist, besteht darin, dass seit der Antike bis zum heutigen Tage in der Literatur immer wieder behauptet wird, dass es nur dreizehn weitere Archimedische Körper gibt. Der Autor zeigt in der vorliegenden Arbeit, dass der Grund für diese Diskrepanz darin liegt, dass in der Vergangenheit oftmals zwei verschiedene Definitionen nicht konsequent auseinander gehalten wurden. 
distinctions between the two enumerations. It is surprising how errors of this type escape detection for a long time, even though there is frequent mention of the results. One example is provided by the enumeration of 4-dimensional simple polytopes with eight facets, by Brückner [7] in 1909. He replaces this enumeration by that of 3-dimensional "diagrams" that he interpreted as Schlegel diagrams of convex 4-polytopes, and claimed that the enumeration of these objects is equivalent to that of the polytopes. However, aside from several "innocent" mistakes in his enumeration, there is a fundamental error: While to all 4-polytopes correspond 3-dimensional diagrams, there is no reason to assume that every diagram arises from a polytope. At the time of Brückner's paper, even the corresponding fact about 3-polyhedra and 2-dimensional diagrams had not yet been established - this followed only from Steinitz's characterization of complexes that determine convex polyhedra [45], [46]. In fact, in the case considered by Brückner, the assumption is not only unjustified, but actually wrong: One of Brückner's polytopes does not exist, see [25]. Other examples of a similar nature involve the enumeration of types of isohedral or isogonal tilings of the plane. In many works, the tilings in question were replaced - for purposes of enumeration - by labeled or marked tilings, or by pairs consisting of a tiling and a group of symmetries. However, the results were erroneously claimed to represent classifications of the tilings proper. The literature is too numerous to be adequately quoted here; the reader should consult Chapters 6 to 9 of [24].

This brings us to the actual topic of this paper. Polyhedra have been studied since antiquity. It is, therefore, rather surprising that even concerning some of the polyhedra known since that time there is a lot of confusion, regarding both terminology and essence. But even more unexpected is the fact that many expositions of this topic commit serious mathematical and logical errors. Moreover, this happened not once or twice, but many times over the centuries, and continues to this day in many printed and electronic publications; the most recent case is in the second issue for 2008 of this journal. I will justify this harsh statement soon, after setting up the necessary background. We need first to clarify the enduring confusion in terminology, and then discuss the actual enduring errors.

\section{Archimedean and uniform polyhedra}

Several kinds of polyhedra have made their first appearance in antiquity, and continued to be investigated throughout the ages. Probably best known among these are the five regular polyhedra, also known as Platonic solids. Representatives of these five kinds of convex polyhedra are beautifully illustrated in countless publications, in print and electronic. Over the centuries, there have been many different definitions, all leading to the same set of five polyhedra - although in some cases a sizable grain of salt has to be supplied in order to reach that goal. One old and widely accepted definition is that a convex polyhedron is regular provided all its faces are congruent regular polygons, meeting in the same number at every vertex. A more recent definition stipulates that a convex polyhedron is regular provided the set of isometric symmetries of the polyhedron acts transitively on the family of all flags. (A flag is a triplet consisting of a face, an edge, and a vertex, all mutually incident). Although the former definition relies on strictly local conditions and the latter one on global properties, the remarkable fact is that they determine the same five polyhedra; many other definitions do the same. 
Convex Archimedean polyhedra form another well-known family, also illustrated in many venues. They are frequently defined by the following requirement, similar to the first one for Platonic solids:

Local criterion: All faces are regular polygons, and the cyclic arrangement of the faces around each vertex is the same.

In this context "same" is understood to allow mirror images, and "around" to include only faces that are incident with the vertex in question.

In contrast to this "local" definition stands the following "global" one:

Global criterion: All faces are regular polygons, and all vertices form one orbit under isometric symmetries of the polyhedron.

Both definitions obviously include Platonic polyhedra, as well as regular-faced prisms and antiprisms of arbitrarily many sides. However, many writers specify that the polyhedra just mentioned should (by fiat, and because of tradition) be excluded and that by "polyhedra satisfying the local (or the global) criterion" we should understand only those that are neither regular, nor prisms or antiprisms. For simplicity of exposition, in what follows we shall accede to this view even though it lacks any logical basis.

A lot of confusion surrounding the topic is the result of inconsistent terminology. Many writers call Archimedean those polyhedra that satisfy the local criterion, and many call uniform or semiregular the ones that satisfy the global criterion. However, others give the name Archimedean polyhedra to those satisfying the global definition. Still other writers (such as Walsh [50] or Villarino [49]) consider "Archimedean" and "semiregular" as denoting the same polyhedra, as specified by the local definition. Now, since there are two differing definitions it is reasonable to give the two classes of polyhedra different names. If this is accepted, then the polyhedra satisfying the local criterion should be called Archimedean, since it is a stretch to impute to Archimedes an approach via groups of symmetries - as a matter of historical fact, before the nineteenth century nobody was thinking of groups, least of all in geometry. The polyhedra satisfying the global criterion should be called uniform or semiregular.

The lack of standardization of terminology would be only a matter of pedantic hairsplitting if it were not for the following two facts. First, "Archimedean" is the term used most frequently, even though many writers using it do not specify what they mean by "Archimedean polyhedra" (or by polyhedra designated by any of the other names). In the present paper we shall consistently use Archimedean to denote polyhedra satisfying the local criterion.

The second fact is much more important. One might expect that - in analogy to the situation concerning regular polyhedra - the local and global definitions yield the same polyhedra. If this were so, there would be not much of point in insisting on different names; no confusing arises in the case of the regular polyhedra. However, this coincidence does not occur. With our understandings and exclusions, there are fourteen "Archimedean" convex polyhedra (that is, those that satisfy the local criterion), but only thirteen "uniform" (or "semiregular") that satisfy the global criterion. Representatives of the thirteen uniform convex polyhedra are shown in the sources mentioned above, while the fourteenth 


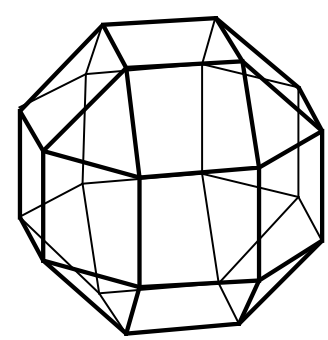

(a)

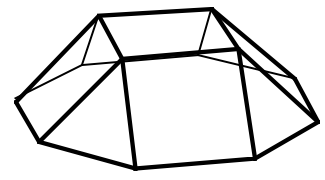

(c)

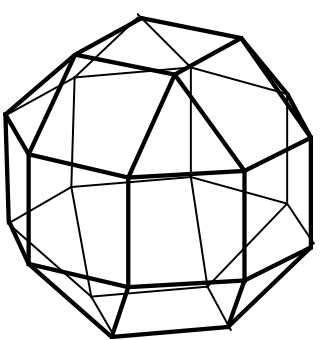

(b)

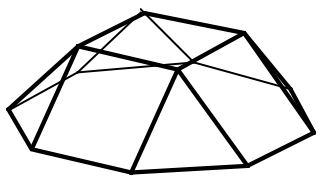

(e)

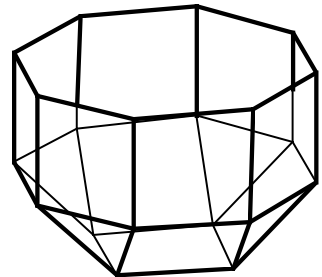

(d)

Fig. 1 (a) The Archimedean (and uniform) rhombicuboctahedron (3.4.4.4), and (b) the Archimedean but non-uniform pseudorhombicuboctahedron. In (d) is shown the common bottom part of the two polyhedra, while (c) and (e) show how they differ in the top part - by a twist of $\pi / 4=45^{\circ}$. The top four vertices (and the bottom four) of the pseudorhombicuboctahedron are not equivalent by symmetries of the polyhedron to the middle eight vertices.

polyhedron is illustrated in Fig. 1. It satisfies the local criterion but not the global one, and therefore is - in our terminology - Archimedean but not uniform. The history of the realization that the local criterion leads to fourteen polyhedra will be discussed in the next section; it is remarkable that this development occurred only in the 20th century. This implies that prior to the twentieth century all enumerations of the polyhedra satisfying the local criterion were mistaken. Unfortunately, many later enumerations make the same error.

It may seem that the confusion of terminology is a matter of little importance. Unfortunately, it is mirrored in surprisingly many writings in which the definitions of Archimedean and uniform polyhedra are conflated, by adopting the local criterion as definition but claiming that global symmetry results. In the sequel, this will be referred to as the enduring error. An unexpected aspect of this mathematical error is that it has been committed by many well-known mathematicians and other scientists. It gives me no pleasure to cite names, but obviously a claim as serious as the one I just made has to be documented. This occurs in Section 4. I should also mention that I have found no indication, in any of the three reviewing journals (Jahrbuch über die Fortschritte der Mathematik, Zentralblatt für Mathematik, Mathematical Reviews), that a reviewer of any of the papers in question was even aware of the problem. A search of "Uniform polyhedra" on Google ${ }^{\mathrm{TM}}$ in 2005 
yielded "about 13,600" results. I checked the first three, namely [37], [51], [27], and found that all three committed this error. A search repeated in June 2008 yielded "about 90,100" items. The first four included [37], [51] and [27], but second on the list was [52] - which did not make the error. Details will be given in Section 4. The printed literature contains many books and articles that do not commit the enduring error, and the same is true for the Web. But my point is that in both kinds of publications, many are guilty of it. It should also be mentioned that many of the same sources contain a lot of other information, often quite valuable.

In the interest of full disclosure I should mention that a version of this paper was submitted some time ago to a different journal. The editor rejected the manuscript, because a referee stated that there are many correct expositions on the Internet. Following this peculiar logic you should not worry about the counterfeit banknote you found in your wallet since there are many genuine ones in circulation.

It is possible - maybe even probable - that the problem of incorrectly enumerating Archimedean polyhedra started with Archimedes. His writings have not survived, and we have only Pappus' word [48] that Archimedes found "the thirteen polyhedra". (The reference to Heron in [31, p. 327] appears to be in error.) But it is clear that if Archimedes used the local definition - as has been believed throughout history - then he should have found "the fourteen polyhedra". The first available enumeration, close to two millennia after Archimedes', was that of Kepler [32], who independently discovered and described thirteen polyhedra which have since been repeatedly rediscovered. Kepler used the local definition as well, hence committed the "enduring error". To Kepler's possible credit it should be said that on one occasion [33, p. 11] he stated without explanation that there are fourteen Archimedean polyhedra; see also Coxeter [10], Malkevitch [38, p. 85]. As far as is known, Kepler never publicly reconciled this statement with his detailed enumeration.

\section{The pseudorhombicuboctahedron}

The first appearance - beyond the fleeting glimpse that Kepler may have had - of the fourteenth polyhedron, usually called the pseudorhombicuboctahedron, happened in a paper by Sommerville [43] in 1905. However, on the face of it this paper deals only with maps in 2-dimensional elliptic, Euclidean and hyperbolic spaces. It has been mentioned in several places (see, for example, [29]) that Sommerville at best has a map that can be interpreted as a Schlegel diagram of the pseudorhombicuboctahedron. However, a more careful reading of his paper (in particular, middle of p. 725), shows that he did actually have polyhedra in mind when discussing the diagrams.

Unfortunately, Sommerville's paper appears to have been completely forgotten for more than a quarter century. The next explicit mention of the pseudorhombicuboctahedron, discovered by J.C.P. Miller, is in [9, p. 336] in 1930. It has at times been called "Miller's mistake", because Miller allegedly intended only to make a model of the rhombicuboctahedron. Miller's polyhedron received wider exposure in Ball and Coxeter [4] and in Fejes Tóth [16, p. 111]; in both books the distinction between local and global properties is stressed.

Independently of Miller, the pseudorhombicuboctahedron was discovered by V.G. Ashkinuse in 1957 [2]. This was mentioned in Ashkinuse [3], and in Lyusternik [35]. A very 
strange presentation of Ashkinuse's polyhedron is in Roman [40]. While in Chapter 5 of [40] a detailed proof is presented of the "fact" that there are precisely thirteen polyhedra that satisfy the local criterion, in Chapter 6 is given a description of both the rhombicuboctahedron and the pseudorhombicuboctahedron. Roman correctly stresses their differences in regard to symmetries but apparently believes that they are isomorphic and should not be counted separately.

\section{The enduring error}

Skipping many early enumerations, I will mention only a few instances from more recent times where the enduring error has been committed in one form or another.

Badoreau [5] follows the global approach, and justly criticizes a well-known work of Catalan [8] for various errors; however, he does not observe that Catalan's local approach is incomplete and is not equivalent to his own global one.

Lines [34] devotes Chapter 12 to Archimedean polyhedra (as defined by the local criterion), and "proves" that there are precisely thirteen of them; this is the source on which Cundly and Rollett [13] base the presentation in their well-known book. In his deservedly popular book, Wenninger [53, p. 2] states "Archimedean or semi-regular solids ... have regular polygons as faces and all vertices equal but admit a variety of such polygons in one solid. There are thirteen such solids ...". Wenninger then gives as reference the book by Lines [34] just mentioned - even though it is clear that Wenninger is really concerned with polyhedra that satisfy the global criterion. Wenninger's book is quoted as the source in some "college geometry" texts (for example, Sibley [41, p. 55]). Lines [34] is also mentioned as a source for the definition of "Archimedean or semiregular polyhedra" by Villarino [49].

Fejes Tóth [15, p. 18] lists the thirteen Archimedean polyhedra as defined by the local criteria, quoting Brückner [6] as authority. This refers to Sections 99 and 106, pp. 121-122 and 132-133, of Brückner's book, where references to several earlier enumerations can be found. It should be noted that while Brückner seems to have had uniform polyhedra in mind, his definitions are local, although different from ours. He never mentions the necessity of investigating whether any of the combinatorial possibilities leads to more than a single polyhedron. (It is strange that the second edition of [15] repeats the error of the first edition mentioned above, although the situation is correctly presented in [16].) Williams [55] and Field [17] assert that local and global definitions yield the same polyhedra. Gerretsen and Verdenduin [18, p. 277] find only thirteen Archimedean polyhedra, although their definitions (different from the one accepted here) in fact allow more than fourteen. Peterson's statements in [39] concerning regular and Archimedean polyhedra are confused and incorrect in several respects.

Maeder [36], [37] states "Uniform polyhedra consist of regular faces and congruent vertices. Allowing for non-convex faces and vertex figures, there are 75 such polyhedra, as well as 2 infinite families of prisms and antiprisms." This is a typical "enduring error", since in the context of more general polyhedra considered by Maeder the correct number for polyhedra satisfying the local criteria he uses is at least 77 . The second non-uniform one (first described in [30]; see also [29]) is shown in Fig. 2(c). In part (a) is shown 


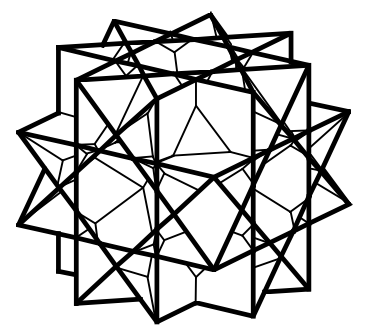

(a)

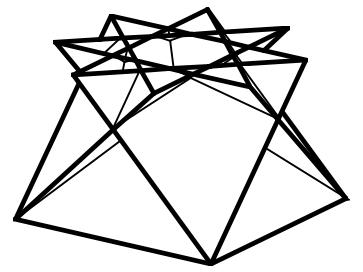

(d)

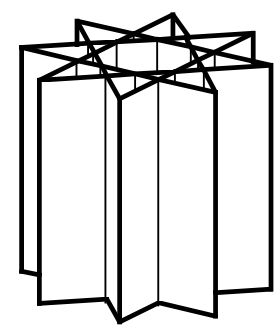

(b)

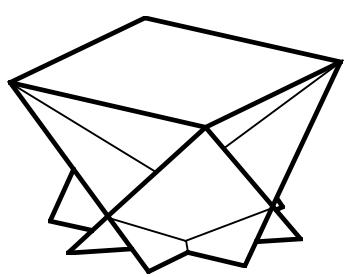

(e)

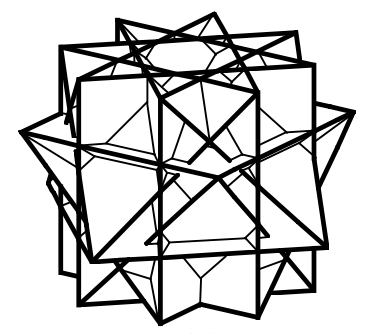

(c)

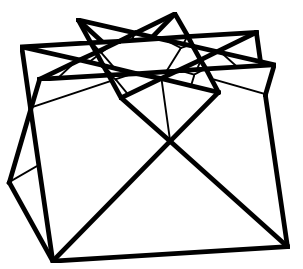

(f)

\footnotetext{
Fig. 2 The Archimedean (and uniform) quasirhombicuboctahedron (3.4.4.4) is shown in (a), and the Archimedean but non-uniform pseudoquasirhombicuboctahedron is shown in (c). Part (b) shows the common octagrammatic mantle, and (e) the common part adjacent to the bottom octagram. Parts (d) and (f) are the top parts of the two polyhedra; as is visible, they differ by a rotation of $\pi / 4=45^{\circ}$.
}

the quasirhombicuboctahedron (see p. 132 of [53]) and in (b), (d) and (e) a certain partition of the set of its faces. At each vertex there are three squares and one triangle, as in the rhombicuboctahedron shown in Fig. 1, but their disposition in space is different. We note that with the global definition, 75 uniform polyhedra (other than prisms and antiprisms) are described in [11], with no claim that the enumeration is complete. (These 75 include the five Platonic and thirteen convex uniform polyhedra, as well as the four Kepler-Poinsot regular non-convex ones. The omission of the five Platonic polyhedra from Theorem 1 of [49] is in contradiction to the definitions given - although this may be deemed an "innocent" error.) All 75 are illustrated in [11], [53], and [26] and in many websites, such as [27], [37], [52]. The completeness of this enumeration was established independently by [44], [42] and [47].

Weisstein [51] in the MathWorld encyclopedia makes a different error, by stating: "The uniform polyhedra are polyhedra with identical polyhedron vertices". This clearly does not imply that the faces are regular polygons - any rectangular box satisfies this definition. Even if "identical" is understood as "equivalent under symmetries of the polyhedron" (and not as the more natural and more general interpretation that the vertices have congruent neighborhoods), then this is precisely the definition of "isogonal polyhedra". It is wellknown that isogonal polyhedra exist in a virtually inexhaustible variety of types (see [21]), and not just 75 as claimed. There are many other errors on this site. 
In the companion article [51] Weisstein writes: "The 13 Archimedean solids are the convex polyhedra that have a similar arrangement of non-intersecting regular convex polygons of two or more different types arranged in the same way about each vertex with all sides the same length." This is precisely the "enduring error"; the final part "all sides the same length" is clearly superfluous.

A special mention should be made of several sources which are knowledgeable and authoritative in the matters under discussion, but contribute to the confusion by a different route, that is misleading and constitutes a logical error. They define the polyhedra they consider by the local criterion, state that their enumeration leads to the thirteen uniform ones, and then - later - observe that this is actually not so, but that the additional polyhedron can be excluded by some supplementary requirement.

Cromwell [12] describes the pseudorhombicuboctahedron, but does not include it among the Archimedean ones (which he defines by the local criterion, see [12, p. 79]). Cromwell's peculiar argument is that to deserve the name "Archimedean" polyhedra must possess properties of global symmetry, that is, be uniform. To quote (from p. 91 of [12]):

... some writers have suggested that this polyhedron [the pseudorhombicuboctahedron] should be counted as a fourteenth Archimedean solid. This, however, misses the point. The true Archimedean solids, like the Platonic solids, have an aesthetic quality which Miller's solid does not possess. This attractiveness comes from their high degree of symmetry - a property that is easily appreciated and understood on an intuitive level. It is not the congruence of the solid angles that is the important characteristic but rather the fact that the solid angles are all indistinguishable from one another.

The present paper is not an appropriate place to debate the aesthetics of polyhedra. One important point to be made here is that the definition as accepted (but later disavowed) yields fourteen polyhedra; the decision to exclude one on other grounds should not be made after claiming that only thirteen polyhedra meet the original requirements. It may also be noted that even if one were to accept Cromwell's restricted concept of Archimedean polyhedra, the many authors who missed the pseudorhombicuboctahedron were not enumerating aesthetically appealing polyhedra. They were looking for polyhedra that satisfy the local condition of "congruence of solids angles" - and made an indisputable error in that enumeration. A moot point is the apparent claim that in the pseudorhombicuboctahedron the solid angles are not indistinguishable; how can they be distinguished seeing that they are congruent?

The presentation in Hart [27] is similar to Cromwell's, but at even greater remove. Uniform polyhedra are defined by the local criterion, and the "enduring error" is evident by the statement that there are 75 of them, although in the present context the number is at least 77. There is a link to [28], which deals with convex polyhedra and contains an explicit statement that there are just thirteen satisfying the local criteria. The polyhedra are listed by symbols and names, with links to impressive illustrations. However, after this, there is an "Exercise" stating:

Just saying that the same regular polygons appear in the same sequence at each vertex is not a sufficient definition of these polyhedra. The Archimedean solid 
(shown at right) in which three squares and a triangle meet at each vertex is the rhombicuboctahedron. Look at it, and then imagine another, similar, convex solid with three squares and an equilateral triangle at each vertex.

The answer gives a link to [29], where the pseudorhombicuboctahedron is shown and its history is discussed. Following this link, Hart [28] states:

A more precise definition of these Archimedean solids would be that [they] are convex polyhedra composed of regular polygons such that every vertex is equivalent.

For this replacement of the local criterion by the global one Hart [29] gives the same argument as Cromwell, by saying:

The pseudo-rhombicuboctahedron is not classified as a semi-regular polyhedron because the essence (and beauty) of the semi-regular polyhedra is not about local properties of each vertex, but the symmetry operations under which the entire object appear unchanged.

Which is a correct argument for not including the pseudorhombicuboctahedron among uniform (or semiregular) polyhedra, but does not excuse its exclusion from the "Archimedean" polyhedra as defined in [28] by the local criterion.

Similar in spirit is the discussion in Kappraff [31, Ch. 9]. The Archimedean polyhedra (called there "semiregular") are defined by the local criterion, and it is stated that Archimedes discovered thirteen polyhedra of this kind. The existence of a fourteenth "semiregular" polyhedron is mentioned later, but it is not counted as an Archimedean polyhedron because it fails to have the following property [31, p. 328]:

Archimedes' original 13 polyhedra can be inscribed in a regular tetrahedron so that four appropriate faces share the faces of a regular tetrahedron ... This distinguishes them from prisms and antiprisms ... and from ... the pseudorhombicuboctahedron...

Although this property of Archimedean solids is interesting, there is no indication in the literature that Archimedes had any such property in mind - just as there is no indication he considered symmetry groups as operating on the polyhedra in question.

The reason for the error of missing one of the Archimedean polyhedra by all the authors that were actually enumerating them (and not just quoting other writers) is due to an error in the logic of enumeration. How could such an error arise, and be perpetuated by being repeated over and over again? Only through the neglect of rules to which we all profess to adhere, but in practice often fail to follow.

To see this, let us recall the general procedure of determining all convex Archimedean polyhedra. One first draws up a list of cycles of faces around a vertex that are possible candidates for cycles of faces of Archimedean polyhedra. The precise steps of compiling such lists differ from author to author; they rely either on the fact that the sum of angles of all faces incident to a vertex of a convex polyhedron is less than $2 \pi=360^{\circ}$, or on Euler's theorem, or on some other considerations. Using various arguments, the starting list is pared down to one that consists of precisely thirteen different cycles (besides those that correspond to regular polyhedra, to prisms or to antiprisms). Showing that each of 
these thirteen cycles actually corresponds to a convex polyhedron with regular faces then completes the enumeration.

However, as anybody who tries to enumerate any sort of objects knows, if you wish to get all the objects it is not enough to get a list of candidates and determine which among them are actually realizable as objects of the desired kind. You also have to find out if any candidate can be realized by more than one object. Unfortunately, over the centuries, none of the geometers that dealt with Archimedean polyhedra bothered with this last task. As shown in Fig. 1, in one of the cases a cycle corresponds to two distinct polyhedra, raising the number of Archimedean polyhedra to fourteen.

What is the moral of this story? Actually, there are several. First, define precisely the objects you wish to consider, and stick consistently with the definition. Second, when carrying out enumerations, be sure you do not miss any of the objects. (Be also sure you do not count any twice!) Third, and possibly most importantly, when quoting some results from the literature, apply common-sense precautions: Make sure that you understand the definitions, verify the claims, and check the deductions.

In reality, if some "fact" is "well-known", one is often inclined to let one's guard down. Put differently, when the result is a foregone conclusion, logical niceties get the short shrift in what amounts to "wishful seeing". Unfortunately, that is how many errors are propagated in the literature. The "fact" that there are precisely thirteen Archimedean polyhedra is a prime example of such a failure of our critical processes. Another illustration of the same kind of error - turning a blind eye to facts that do not support the evidence or contradict it appears in the famous and often reprinted work of Weyl [54]. Since he knows that any net (map) on a sphere with only meshes (faces) of at most six sides cannot have only hexagonal meshes, in his desire to bolster this claim Weyl produces several examples (Figs. 51, 52 and 55 of [54]) of spherical meshes. After each, he points out the existence of pentagons. However, his claims are logically invalid, since each of these nets contains heptagons. It is not relevant to the critique that, in fact, the presence of heptagons increases the number of pentagons that must be present.

With more general definitions of polygons and polyhedra (such as studied in [22] and [23]) it is easy to find many additional examples of polyhedra that satisfy the local - but not the global - criteria. However, one interesting question remains unsolved:

Are there any additional Archimedean but not uniform polyhedra in the class of polyhedra admitted by the definition in [11]?

It may be conjectured that the answer is negative, but a proof of this is probably quite complicated.

Added in proof. Another recent example of the "enduring error" occurs in the book Euler's Gem by David S. Richeson (Princeton University Press 2008), pages 49 and 57. Richeson defines polyhedra he calls Archimedean or semiregular by the local criterion, and asserts that there are precisely thirteen of them (besides the regular polyhedra, prisms, and antiprisms). 


\section{References}

[1] Andreini, A.: Sulle reti di poliedri regolari e semiregolari e sulle corrispondenti reti correlative. Mem. Società Italiana della Scienze 14 (1905) 3, 75-129.

[2] Ashkinuse, V.G.: On the number of semiregular polyhedra. [In Russian] Matemat. Prosveshch. 1957, no. $1,107-118$.

[3] Ashkinuse, V.G.: Polygons and polyhedra. [In Russian] In: Enciklopediya elementarnoi matematki. Vol. 4. Gostehizdat, Moscow 1963. German translation: Aschkinuse, W.G.: Vielecke und Vielflache, 391-456. In: Alexandroff, P.S.; Markuschewitsch, A.I.; Chintschin, A.J. (eds.): Enzyklopädie der Elementarmathematik. Vol. 4 (Geometrie). VEB Deutscher Verlag der Wissenschaften, Berlin 1969.

[4] Ball, W.W.R.; Coxeter, H.S.M.: Mathematical Recreations and Essays. $11^{\text {th }}$ ed. Macmillan, London 1939. $12^{\text {th }}$ ed. Univ. of Toronto Press, 1973. $13^{\text {th }}$ ed. Dover, New York 1987.

[5] Badoureau, A.: Mémoire sur les figures isoscèles. J. École Polytech. 30 (1881), 47-172.

[6] Brückner, M.: Vielecke und Vielflache. Theorie und Geschichte. Teubner, Leipzig 1900.

[7] Brückner, M.: Ueber die Ableitung der allgemeinen Polytope und die nach Isomorphismus verschiedenen Typen der allgemeinen Achtzelle (Oktatope). Verh. Nederl. Akad. Wetensch. Afd. Natuurk. Sect. 1, V. 10, No. 1 (1909), 27 pp. +2 plates.

[8] Catalan, E.C.: Mémoire sur la théorie des polyèdres. J. École Polytech. 24 (1865), 1-71.

[9] Coxeter, H.S.M.: The polytopes with regular-prismatic vertex figures I. Phil. Trans. Roy. Soc. London, Ser. A, 229 (1930), 329-425.

[10] Coxeter, H.S.M.: Kepler and mathematics. In: Beer, A.; Beer, P. (eds.): Four Hundred Years. Proc. Conference held in Honour of Johannes Kepler. Vistas in Astronomy, Vol. 18. Pergamon Press, Oxford 1974, 661-670.

[11] Coxeter, H.S.M.; Longuet-Higgins, M.S.; Miller, J.C.P.: Uniform polyhedra. Philos. Trans. Roy. Soc. London, Ser. A, 246 (1953/54), 401-450.

[12] Cromwell, P.R.: Polyhedra. Cambridge Univ. Press 1997.

[13] Cundy, H.M.; Rollett, A.P.: Mathematical Models. Clarendon Press, Oxford 1952, $2^{\text {nd }}$ ed. 1961.

[14] Daublebsky von Sterneck, R.: Die Configurationen 123. Monatsh. Math. Phys. 6 (1895), 223-255 + 2 plates.

[15] Fejes Tóth, L.: Lagerungen in der Ebene, auf der Kugel und im Raum. Springer, Berlin 1953. $2^{\text {nd }}$ ed. 1972.

[16] Fejes Tóth, L.: Reguläre Figuren. Akadémiai Kiadó, Budapest 1965. English translation: Regular Figures. Macmillan, New York 1964.

[17] Field, J.V.: Rediscovering the Archimedean polyhedra: Piero della Francesca, Luca Pacioli, Leonardo da Vinci, Albrecht Dürer, Daniele Barbaro and Johannes Kepler. Arch. Hist. Exact Sci. 50 (1997), 241-289.

[18] Gerretsen, J.; Verdenduin, P.: Polygons and polyhedra. In: Behnke, H. et al. (eds.): Fundamentals of Mathematics. Vol. 2 (Geometry). Chapter 8. MIT Press, Cambridge, MA 1974. $2^{\text {nd }}$ ed. 1983. Translation of the German original Grundzüge der Mathematik. Vandenhoeck \& Ruprecht, Göttingen 1967.

[19] Gropp, H.: On the existence and nonexistence of configurations $n_{k}$. J. Combin. Inform. System Sci. 15 (1990), 34-48.

[20] Grünbaum, B.: Uniform tilings of 3-space. Geombinatorics 4 (1994), 49-56.

[21] Grünbaum, B.: Isogonal prismatoids. Discrete Comput. Geom. 18 (1997), 13-52.

[22] Grünbaum, B.: Are your polyhedra the same as my polyhedra? In: Aronov, B.; Basu, S.; Pach, J.; Sharir, M. (eds.): Discrete and Computational Geometry: The Goodman-Pollack Festschrift. Springer, New York 2003, 461-488.

[23] Grünbaum, B.: "New” uniform polyhedra. Discrete Geometry: In Honor of W. Kuperberg's 60th Birthday. Monographs and Textbooks in Pure and Applied Mathematics, Vol. 253. Marcel Dekker, New York 2003, $331-350$. 
[24] Grünbaum, B.; Shephard, G.C.: Tilings and Patterns. Freeman, New York 1987.

[25] Grünbaum, B.; Sreedharan, V.P.: An enumeration of simplicial 4-polytopes with 8 vertices. J. Combin. Theory 2 (1967), 437-465.

[26] Har'El, Z.: Uniform solutions for uniform polyhedra. Geom. Dedicata 47 (1993), 57-110.

[27] Hart, G.W.: Uniform polyhedra. http://www.georgehart.com/virtual-polyhedra/uniform-info.html

[28] Hart, G.W.: Archimedean polyhedra. http://www.georgehart.com/virtual-polyhedra/archimedean-info.html

[29] Hart, G.W.: Pseudorhombicuboctahedra. http://www.georgehart.com/virtual-polyhedra/pseudo-rhombicuboctahedra.html

[30] Hughes Jones, R.: The pseudo-great rhombicuboctahedron. Math. Sci. 19 (1994) 1, 60-63.

[31] Kappraff, J.: Connections. The Geometric Bridge Between Art and Science. McGraw-Hill, New York $1991,2^{\text {nd }}$ ed. World Scientific, Singapore 2001.

[32] Kepler, J.: The Harmony of the World. Mem. Amer. Philos. Soc. 209 (1997). Translation of the Latin original published in 1619.

[33] Kepler, J.: The Six-Cornered Snowflake. Clarendon Press, Oxford 1966. Translation of the Latin original published in 1611 .

[34] Lines, L.: Solid Geometry. Dover, New York 1965.

[35] Lyuesternik, L.A.: Convex Figures and Polyhedra. [In Russian] English translation by T.J. Smith, Dover, New York 1963, and by D.L. Barnett, Heath, Boston 1966.

[36] Maeder, R.E.: Uniform polyhedra. The Mathematica Journal 3 (1993) 4, 48-57.

[37] Maeder, R.E.: Uniform Polyhedra. Excerpt of Chapter 9 of R. Maeder's book The Mathematica Programmer II. http://www.mathconsult.ch/showroom/unipoly/

[38] Malkevitch, J.: Milestones in the history of polyhedra. In: Senechal, M.; Fleck, G. (eds.): Shaping Space: A Polyhedral Approach. Birkhäuser, Boston 1988, 80-92.

[39] Peterson, I.: Polyhedron man: Spreading the word about the wonders of crystal-like geometric forms. Science News 160 (2001), 396-398.

[40] Roman, T.: Reguläre und halbreguläre Polyeder. VEB Deutscher Verlag der Wissenschaften, Berlin 1968.

[41] Sibley, T.Q.: The Geometric Viewpoint. A Survey of Geometries. Addison-Wesley, Reading, MA 1998.

[42] Skilling, J.: The complete set of uniform polyhedra. Philos. Trans. Roy. Soc. London, Ser. A, 278 (1975), 111-135.

[43] Sommerville, D.M.Y.: Semi-regular networks of the plane in absolute geometry. Trans. Roy. Soc. Edinburgh 41 (1905), 725-747, with 12 plates.

[44] Sopov, S.P.: Proof of the completeness of the enumeration of uniform polyhedra. [In Russian] Ukrain. Geom. Sb. 8 (1970), 139-156.

[45] Steinitz, E.: Polyeder und Raumeinteilungen. Enzykl. Math. Wiss., Vol. 3 (Geometrie) Part 3AB12, 1922, 1-139.

[46] Steinitz, E.; Rademacher, H.: Vorlesungen über die Theorie der Polyeder. Springer, Berlin 1934.

[47] Szepesvári, I.: On the number of uniform polyhedra. I, II. [In Hungarian] Mat. Lapok 29 (1977/81), 273328.

[48] Ver Eecke, P.: Pappus d'Alexandrie: La collection mathématique. Desclée, de Brouwer, Paris 1933.

[49] Villarino, M.B.: On the Archimedean or semiregular polyhedra. Elem. Math. 63 (2008), $76-87$.

[50] Walsh, T.R.S.: Characterizing the vertex neighbourhoods of semi-regular polyhedra. Geom. Dedicata 1 (1972), 117-123.

[51] Weisstein, E.W.: Uniform Polyhedron. From MathWorld - A Wolfram Web Resource. http://mathworld.wolfram.com/UniformPolyhedron.html 
[52] Wikipedia: Uniform polyhedron. http://en.wikipedia.org/wiki/Uniform-polyhedron

[53] Wenninger, M.J.: Polyhedron Models. Cambridge Univ. Press 1971.

[54] Weyl, H.: Symmetry. Princeton Univ. Press 1952. Reprinted 1982, 1989.

[55] Williams, R.: Natural Structure. Eudæmon Press, Moorpark, CA, 1972. Reprint: The Geometrical Foundation of Natural Structures. Dover, New York 1979.

Branko Grünbaum

Department of Mathematics

University of Washington

Box 354350

Seattle, WA 98195, USA

e-mail: grunbaum@math.washington.edu 